\title{
The Computation of Flow and Heat Transfer through an Orthogonally Rotating Square-Ended U-Bend Using Low-Reynolds-Number Models
}

\author{
Konstantinos-Stephen P. Nikas \\ Laboratory of Aerodynamics, Department of Mechanical Engineering, National Technical University of Athens (NTUA), \\ 9 Heroon Polytexneiou Street, P.O. Box 64070, Zografou Campus, 15773 Athens, Greece \\ Email: mcjtpn4@fluid.mech.ntua.gr \\ Hector lacovides \\ School of Mechanical, Aerospace and Civil Engineering, The University of Manchester, Sackville Street, \\ P.O. Box 88, Manchester M60 1QD, UK \\ Email: h.iacovides@manchester.ac.uk \\ Received 22 February 2004
}

\begin{abstract}
We present computations of heat and fluid flow through a square-ended U-bend that rotates about an axis normal to both the main flow direction and also the axis of curvature. Two-layer and low-Reynolds-number mathematical models of turbulence are used at effective-viscosity (EVM) level and also at second-moment-closure (DSM) level. Moreover, two length-scale correction terms to the dissipation rate of turbulence are used with the low-Re models, the original Yap term, and a differential form that does not require the wall distance (NYap). The resulting predictions are compared with available flow and heat transfer measurements of water. While the main flow features are well reproduced by all models, the development of the mean flow within and just after the bend is better reproduced by the low-Re models. Turbulence levels within the rotating U-bend are underpredicted, but DSM models produce a more realistic distribution. Along the leading side, all models overpredict heat transfer levels just after the bend. Along the trailing side, the heat transfer predictions of the low-Re DSM with the NYap, are close to the measurements.
\end{abstract}

Keywords and phrases: RANS, low-Re models, two-layer models, heat transfer, strong curvature, orthogonal rotation.

\section{INTRODUCTION}

Tight square-ended U-bends, shown in Figure 1, can be consideredas an idealised representation of the tight turns present in internal cooling passages of gas-turbine blades. The flow inside these cooling passages is complex and highly three-dimensional, influenced by the presence of the sharp U-bends, surface rib roughness, and also by the rotation of the blades. The axis of rotation is normal to the main flow direction, known as orthogonal rotation, and in relation to the U-bend, its orientation tends to be closer to that shown in Figure 1a where it is normal to the axis of curvature. The optimisation of such passages could greatly benefit from the use of numerical flow solvers that can reliably simulate the flow and thermal development within them. The development of such solvers requires the use of mathematical models

This is an open access article distributed under the Creative Commons Attribution License, which permits unrestricted use, distribution, and reproduction in any medium, provided the original work is properly cited. of turbulence that can reproduce the effects of strong curvature, rib roughness, and rotation on the turbulence field. In this contribution, we look at the numerical simulation of flows that contain two of the three main features present in blade cooling passages, namely strong curvature and orthogonal rotation.

Flow and heat transfer through tight U-bends, with and without rotation, have been the subject of a number of recent experimental and numerical investigations such as those of Ekkad and Han [1] and Rigby et al. [2]. In the authors' group, previous numerical investigations and most earlier experimental studies focused on round-ended U-bends, shown in Figure 1b, see Bo et al. [3] and Iacovides et al. [4]. These studies employed low-Reynolds-number models at both effective-viscosity and second-moment closure levels. They showed that all turbulence models used predicted that separation along the inner wall of the bend separation occurs later than what is indicated by the experimental data, though the introduction of second-moment closures led to considerable improvements. In the case of square-ended U-bend, 
in addition to the strong curvature, the presence of the sharp $90^{\circ}$ corners along the outer wall and the continuous change in cross-sectional area also influence the flow and thermal developments. The recent emergence of local flow and thermal data for square-ended U-bends from our group, Iacovides et al. [5] and also from other groups, provided the validation data necessary to assess the effectiveness of turbulence models under these more complex conditions. As we have recently reported, Nikas and Iacovides [6], in the prediction of flow and heat transfer in stationary square-ended U-bends, effective-viscosity and second-moment closures returned very similar flow predictions, both underpredicting the separation bubble at the bend exit. Some differences were observed in the predictions of wall heat transfer, with the low-Re second-moment closure producing the more reliable simulations.

Here, attention is focused on flow and heat transfer through square-ended U-Bends that rotate orthogonally about an axis normal to that of curvature, as shown in Figure 1a. The objective is to use the same models of turbulence employed in our earlier studies of flows through round- and square-ended U-bends and also through ribbed passages, see Iacovides and Raisee [7], in order to assess their effectiveness in predicting the flow and thermal development in rotating square-ended U-bends. These models include two-layer and low-Re models at both effective-viscosity and secondmoment closure level.

\section{THEORETICAL MODEL}

The flow computations have been obtained through the solution of the Reynolds-averaged flow equations, presented here in Cartesian tensor notation, for a rotating frame of reference:

$$
\begin{aligned}
& \text { continuity: } \frac{\partial}{\partial x_{i}}\left(\rho U_{i}\right)=0, \\
& \begin{aligned}
\text { momentum: } \frac{\partial}{\partial x_{j}}\left(\rho U_{j} U_{i}\right) \\
=-\frac{\partial P}{\partial x_{i}}+\frac{\partial}{\partial x_{j}}\left[\mu\left(\frac{\partial U_{i}}{\partial x_{j}}+\frac{\partial U_{j}}{\partial x_{i}}\right)-\rho \overline{u_{i} u_{j}}\right] \\
\quad-2 \rho \varepsilon_{i j p} \Omega_{p} U_{j}-\rho\left[\Omega_{j} X_{j} \Omega_{i}-\Omega_{j} X_{i} \Omega_{j}\right], \\
\text { energy: } \frac{\partial}{\partial x_{j}}(\rho T)=\frac{\partial}{\partial x_{j}}\left[\frac{\mu}{\operatorname{Pr}} \frac{\partial T}{\partial x_{j}}-\rho \overline{u_{i} t}\right],
\end{aligned}
\end{aligned}
$$

where $\rho$ is the density, $\mu$ the molecular viscosity, $P$ the pressure, $T$ the mean temperature, $U_{i}$ the contravariant velocity components, and $\Omega_{i}$ the angular velocity. In (2), $\Omega_{p}$ denotes the rotation vector of the co-ordinate system and $X_{i}$ the position vector relative to the axis of rotation. The first group of rotation terms in (2) represent the Coriolis forces and the second group the centrifugal forces. The flows computed here are water flows that are either isothermal or involve temperature differences small enough for the density to be practically constant. The centrifugal terms therefore do not influence the flow development.

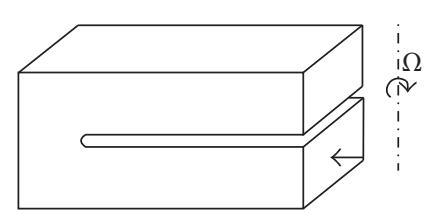

(a)

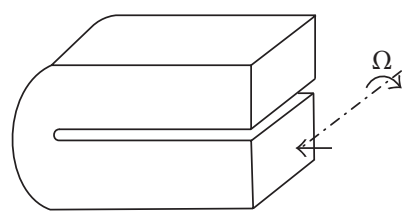

(b)
FIGURE 1: Flow geometries and modes of rotation: (a) square-ended U-bend, and (b) round-ended U-bend.

\subsection{Turbulence modelling}

\subsubsection{Effective-viscosity models}

Two EVM models have been used, a two-layer $k$ - $\varepsilon$ /oneequation model, and a low-Re $k-\varepsilon$ model. In both versions, the Reynolds stresses and the turbulent heat fluxes are obtained from the effective-viscosity and effective-diffusivity approximations, respectively:

$$
\begin{gathered}
\rho \overline{u_{i} u_{j}}=\frac{2}{3} k \delta_{i j}-\mu_{t}\left(\frac{\partial U_{i}}{\partial x_{j}}+\frac{\partial U_{j}}{\partial x_{i}}\right), \\
\overline{u_{i} t}=-\frac{\mu_{t}}{\sigma_{\mathrm{T}}} \frac{\partial T}{\partial x_{i}},
\end{gathered}
$$

where $\overline{u_{i} u_{j}}$ denotes the Reynolds stress tensor, $\overline{u_{i} t}$ the turbulent heat flux, $k$ the turbulent kinetic energy, $\mu_{t}$ the eddy viscosity. The two-layer EVM model consists of the standard high-Re version in the fully turbulent region, matched to Wolfshtein's [8] low-Re one-equation model of $k$-transport in the near-wall regions. This approach allows the resolution of the mean flow across the viscous wall sublayer without the need to use an excessively fine near-wall grid. The equations involved are widely used and are thus not presented here due to space limitations.

The low-Re EVM model employed is Launder-Sharma [9] well-known extension of the high-Re $k-\varepsilon$ that can reproduce the wall damping of turbulence, and hence can be used across the viscous sublayer. Again, the widely used equations involved are not included here for brevity.

\subsubsection{Low-Re DSM models}

The DSM closures employed here are rather simple and empirically derived extensions to the basic DSM model that relies on the linear redistribution terms and use the wallreflection terms. They have evolved from the low-Re ASM closures proposed by Iacovides and Launder [10] and were initially applied to flow and heat transfer through U-Bends of mild curvature. They were subsequently extended by Iacovides and Toumpanakis [11] to low-Re DSM closures and were first used for the computation of turbulent flows through rotating cavities (see Iacovides et al. [12]), where they produced satisfactory predictions. Iacovides and Raisee (see $[7,13])$ have also applied these low-Re DSM closures more recently to the computation of flow and heat transfer through ribbed passages, where again their introduction improved the thermal predictions. More recently, we have 
TABLE 1: Equations for the basic low-Re DSM model.

$$
\begin{gathered}
\frac{\partial}{\partial x_{j}}\left(\rho U_{k} \overline{u_{i} u_{j}}\right)=\frac{\partial}{\partial x_{k}}\left[\left(\mu+\frac{\mu_{t}}{\sigma_{k}}\right) \frac{\partial \overline{u_{i} u_{j}}}{\partial x_{k}}\right]+P_{i j}-\rho \varepsilon_{i j}+\varphi_{i j}-\left[H_{i j}-\frac{1}{3} H_{k k} \delta_{i j}\right]+J_{i j} \\
P_{i j}=-\rho\left(\overline{u_{i} u_{k}} \frac{\partial U_{j}}{\partial x_{k}}+\overline{u_{j} u_{k}} \frac{\partial U_{i}}{\partial x_{k}}\right)-2 \rho \Omega_{p}\left(\varepsilon_{i p q} \overline{u_{q} u_{j}}+\varepsilon_{j p q} \overline{u_{q} u_{i}}\right) \\
\varepsilon_{i j}=\frac{2}{3}\left(1-f_{\varepsilon}\right) \varepsilon \delta_{i j}+f_{\varepsilon} \frac{\overline{u_{i} u_{k}}}{k} \varepsilon \\
\varphi_{i j}=-c_{1} \frac{\varepsilon}{k}\left(\overline{u_{i} u_{j}}-\frac{2}{3} k \delta_{i j}\right)-c_{2}\left(P_{i j}-\frac{2}{3} P_{k} \delta_{i j}\right)+f_{w}\left(\varphi_{i j, 1}^{w}+\varphi_{i j, 2}^{w}\right) \\
\varphi_{i j, 1}^{w}=-c_{1}^{w} \frac{\varepsilon}{k}\left(\overline{u_{k} u_{m}} n_{k} n_{m} \delta_{i j}-\frac{3}{2} \overline{u_{k} u_{i}} n_{k} n_{j}-\frac{3}{2} \overline{u_{k} u_{j}} n_{k} n_{i}\right)\left\{\frac{k^{1.5}}{\varepsilon c_{l} x_{n}}\right\} \\
\varphi_{i j, 2}^{w}=-c_{2}^{w} \frac{\varepsilon}{k}\left(\varphi_{k m 2} n_{k} n_{m} \delta_{i j}-\frac{3}{2} \varphi_{i k 2} n_{k} n_{j}-\frac{3}{2} \varphi_{j k 2} n_{k} n_{i}\right)\left\{\frac{k^{1.5}}{\varepsilon c_{l} x_{n}}\right\} \\
\text { Where } \varphi_{i j 2}=-c_{2}\left(P_{i j}-\frac{2}{3} P_{k} \delta_{i j}\right) \\
H_{i j}=f_{H} \frac{\nu}{k}\left(\overline{u_{i} u_{l}} \frac{\partial \sqrt{k}}{\partial x_{l}} \frac{\partial \sqrt{k}}{\partial x_{j}}+\overline{u_{j} u_{l}} \frac{\partial \sqrt{k}}{\partial x_{l}} \frac{\partial \sqrt{k}}{\partial x_{i}}\right) \\
J_{i j}=f_{J} k\left(\frac{\partial U_{i}}{\partial x_{j}}+\frac{\partial U_{j}}{\partial x_{i}}\right) J_{i j} \\
\overline{u_{i} t}=-\rho c_{T} \frac{k}{\varepsilon} \overline{u_{i} u_{j}} \frac{\partial T}{\partial x_{j}}
\end{gathered}
$$

also used these models in the computation of heat and fluid flow through stationary U-bends with encouraging results (see Nikas and Iacovides [6]). The low-Re terms, constants, and damping functions have been determined with reference to fully developed pipe flow and have not been changed in any of the subsequent applications.

Instead of the effective-viscosity approximation (4), the turbulent stresses are now obtained through the solution of separate transport equations, represented by (6) located in Table 1. The transport of the turbulent stresses and also that of the dissipation rate $\varepsilon$, due to turbulent mixing, is modelled through the effective diffusivity concept. The term $P_{i j}$ denotes the generation rate of the turbulent stresses and is obtained through the exact expression given in (7). It is worth noting that the Coriolis force directly influences the generation rate of the Reynolds stresses. This effect obviously cannot be taken into account by effective-viscosity models. In the most general case, the centrifugal force would also contribute to the generation rate of the Reynolds stresses. Because, however, the flow considered here is effectively a constant-density flow, the centrifugal terms are left out. The term $\varepsilon_{i j}$ denotes the dissipation rate of the turbulent stresses which, as shown in (8), is assumed to be isotropic when the flow is fully turbulent and proportional to the ratio $\overline{u_{i} u_{j}} / k$ at the wall. The function $f_{\mathcal{E}}$ is zero when the flow is fully turbulent and one at the wall.

The term $\phi_{i j}$, given in (9), represents the redistribution of turbulent energy among the different components of the Reynolds stress tensor due to fluctuations in the pressure and strain fields. The first two terms denote a linear return to isotropy and isotropisation of production, respectively. They are also present in the widely used high-Re version of the
DSM closure. Terms $\phi_{i j 1}^{w}$ and $\phi_{i j 2}^{w}$, given in (10), (11), and (12), are the conventional wall-reflection terms, proposed by Gibson and Launder [14], to model the "wall-echo" part of the pressure-strain correlation. Near solid surfaces, they remove kinetic energy from the fluctuating component normal to the wall and redistribute it in the other two directions. They have been devised for the fully turbulent region of a flow over a plane wall and make use of the wall distance $x_{n}$ and the unit vector normal to the wall $n$.

Within the viscous sublayer, the wall-reflection terms are damped through the function $f_{w}$. Their task within the viscous sublayer is then performed by $\left(H_{i j}-H_{k k} \delta_{i j} / 3\right)$, where $H_{i j}$ is given by (13). The contribution of this term is more extensively discussed by Bo et al. [3]. It represents a relatively simple way of achieving approximately the correct distribution of the Reynolds stresses across the viscosity-affected sublayer. The term $J_{i j}$, given in (14), increases the sensitivity of the model to the effects of low mean flow Reynolds number. The turbulent heat fluxes are obtained through the generalised gradient diffusion hypothesis, given by (15).

\subsubsection{Two-layer DSM closure}

In the fully turbulent region, $\varepsilon$ is obtained from the same equation used in the high-Re $k-\varepsilon$ model:

$$
\frac{\partial}{\partial x_{j}}\left(\rho U_{j} \varepsilon\right)=\frac{\partial}{\partial x_{j}}\left[\left(\mu+\frac{\mu_{t}}{\sigma_{\varepsilon}}\right) \frac{\partial \varepsilon}{\partial x_{j}}\right]+c_{\varepsilon_{1}} \frac{\varepsilon}{k} P_{k}-\rho c_{\varepsilon_{2}} \frac{\varepsilon^{2}}{k}
$$

In the near-wall region, the dissipation rate of the turbulent kinetic energy, $\varepsilon$, is obtained from the wall distance, as in the 
Wolfshtein model [8],

$$
\varepsilon=\frac{k^{3 / 2}}{l_{\varepsilon}}, \quad \text { but with } l_{\varepsilon}=2.55 Y\left[1-\exp \left(-0.263 y^{*}\right)\right] \text {. }
$$

The damping functions that appear in (8), (9), (10), (11), (12), (13), and (14) depend on the dimensionless wall distance $y^{*}$ and have the following expressions:

$$
\begin{gathered}
f_{\varepsilon}=\exp \left(-\frac{y^{*}}{3}\right), \\
f_{w}=\left[1-\exp \left(-0.12 y^{*}\right)\right]\left[1+\exp \left(-0.03 y^{*}\right)\right], \\
f_{J}=0.06 \exp \left(-\frac{y^{*}}{3}\right), \\
f_{H}=\left(10.2+7.5 y^{*}\right) \exp \left(-\frac{y^{*}}{20}\right) .
\end{gathered}
$$

\subsubsection{Low-Re DSM closure}

The dissipation rate equation is the same as that of the lowRe $k-\varepsilon$ model:

$$
\begin{aligned}
\frac{\partial}{\partial x_{j}}\left(\rho U_{j} \tilde{\varepsilon}\right)= & \frac{\partial}{\partial x_{j}}\left[\left(\mu+\frac{\mu_{t}}{\sigma_{\varepsilon}}\right) \frac{\partial \tilde{\varepsilon}}{\partial x_{j}}\right] \\
& +c_{\varepsilon_{1}} \frac{\tilde{\varepsilon}}{k} P_{k}-\rho c_{\varepsilon_{2}} f_{2} \frac{\tilde{\varepsilon}^{2}}{k}+2 \rho v v_{t}\left[\frac{\partial^{2} U_{i}}{\partial x_{j} \partial w_{q}}\right]^{2},
\end{aligned}
$$

where $v$ and $v_{t}$ denote the kinematic and the kinematic eddy viscosity, respectively. The damping function $f_{2}$, which appears in (19), is $f_{2}=1-0.3 \exp \left(-R_{t}^{2}\right)$. As in the LaunderSharma [9] model, $\mu_{t}$ is obtained from $\mu_{t}=\rho c_{\mu} f_{\mu} k^{2} / \tilde{\varepsilon}$, with $f_{\mu}=\exp \left[-3.4 /\left(1+0.02 R_{t}\right)^{2}\right]$. The damping functions that appear in (6), (7), (8), (9), (10), (11), (12), (13), and (14) now depend on the turbulent Reynolds number $R_{t}$ and have the following expressions:

$$
\begin{gathered}
f_{\varepsilon}=\exp \left(-\frac{R_{t}}{8}\right), \\
f_{w}=\left[1-\exp \left(-\frac{R_{t}}{20}\right)\right]\left[1-\exp \left(-\frac{R_{t}}{100}\right)\right], \\
f_{J}=0.06 \exp \left(-\frac{R_{t}}{8}\right), \\
f_{H}=\left(10+2.6 R_{t}\right) \exp \left(-\frac{R_{t}}{20}\right) .
\end{gathered}
$$

Also $f_{\mu}$, which still appears in the stress (6) and $\varepsilon$ transport equations, is now obtained from

$$
f_{\mu}=\exp \left[-\frac{4}{\left(1+0.01 R_{t}\right)^{2}}\right] .
$$

As mentioned earlier, the damping functions shown in (18) and (20), and (21) have been devised with reference to fully developed pipe flows and not specifically for the ribbed passage flows presented in this study.
TABLE 2: Turbulence modelling constants.

\begin{tabular}{ccccccccccc}
\hline$c_{\mu}$ & $\sigma_{k}$ & $\sigma_{\varepsilon}$ & $c_{\varepsilon 1}$ & $c_{\varepsilon 2}$ & $c_{1}$ & $c_{2}$ & $c_{1}^{W}$ & $c_{2}^{W}$ & $c_{T}$ & $\sigma_{T}$ \\
\hline 0.09 & 1 & 1.22 & 1.44 & 1.92 & 1.8 & 0.6 & 0.5 & 0.3 & 0.32 & 0.9 \\
\hline
\end{tabular}

\subsubsection{Length-scale correction terms}

In separated flows, the Launder-Sharma version of the $\varepsilon$ equation returns excessively high levels of near-wall turbulence. To address this problem, Yap [15] proposed the addition of a correction term to the $\varepsilon$ equation, based on the wall distance $Y$,

$$
\text { Yap }=\max \left[0.83 \frac{\tilde{\varepsilon}^{2}}{k}\left(\frac{k^{1.5} / \tilde{\varepsilon}}{2.55 Y}-1\right)\left(\frac{k^{1.5} / \tilde{\varepsilon}}{2.55 Y}\right), 0\right] .
$$

Iacovides and Raisee [7] developed a differential form, by (a) introducing the magnitude of the resultant of the length scale $\left(\ell=k^{3 / 2} / \varepsilon\right)$, gradient vector $D \ell$; and (b) by also taking into account the effects of wall damping on the length scale as expressed by Wolfshtein [8]. This leads to the following correction term NYap:

$$
\begin{gathered}
\text { NYap }=\max \left[c_{Y} F(F+1)^{2} \frac{\rho \varepsilon^{2}}{k}, 0\right], \\
D l=\left\{\left(\frac{d l}{d x_{j}}\right)\left(\frac{d l}{d x_{j}}\right)\right\}^{1 / 2}, \\
F=\frac{\left[D l-\left(d l_{\varepsilon} / d Y\right)\right]}{c_{l}}, \\
\left(\frac{d l_{\varepsilon}}{d Y}\right)=c_{l}\left[1-\exp \left(-B_{\varepsilon} R_{t}\right)\right]+B_{\varepsilon} c_{l} R_{t} \exp \left(-B_{\varepsilon} R_{t}\right),
\end{gathered}
$$

with $c_{l}=2.55$ and $B_{\varepsilon}=0.1069$.

Iacovides and Raisee [7] initially suggested that $c_{Y}$ should have the value of 0.83 , as in the original Yap term, for both the low-Re $k-\varepsilon$ and the low-Re DSM models. Here, following Craft [16], who also adopted this term for his realisable DSM, for the low-Re DSM, $c_{Y}$ is set to 0.5 ; while for the low-Re $k-\varepsilon$, it is left as 0.83 .

The other modelling constants that appear in the preceding equations have the values given in Table 2 .

\section{NUMERICAL ASPECTS}

A three-dimensional nonorthogonal finite-volume solver, stream, has been employed, developed at UMIST, which employs the Cartesian velocity decomposition. A collocated grid is used. The simple algorithm is employed for the calculation of the pressure field, with the Rhie-Chow [17] flux modification. In the case of the DSM models, the apparent viscosity concept is used to prevent numerical oscillations that arise from the explicit presence of the Reynolds stress gradients in the momentum equations. For the convective discretization of all transport equations, a bounded form of the quadratic upstream interpolation scheme (QUICK) was used, proposed by Iacovides [18]. For a given mesh, the DSM 

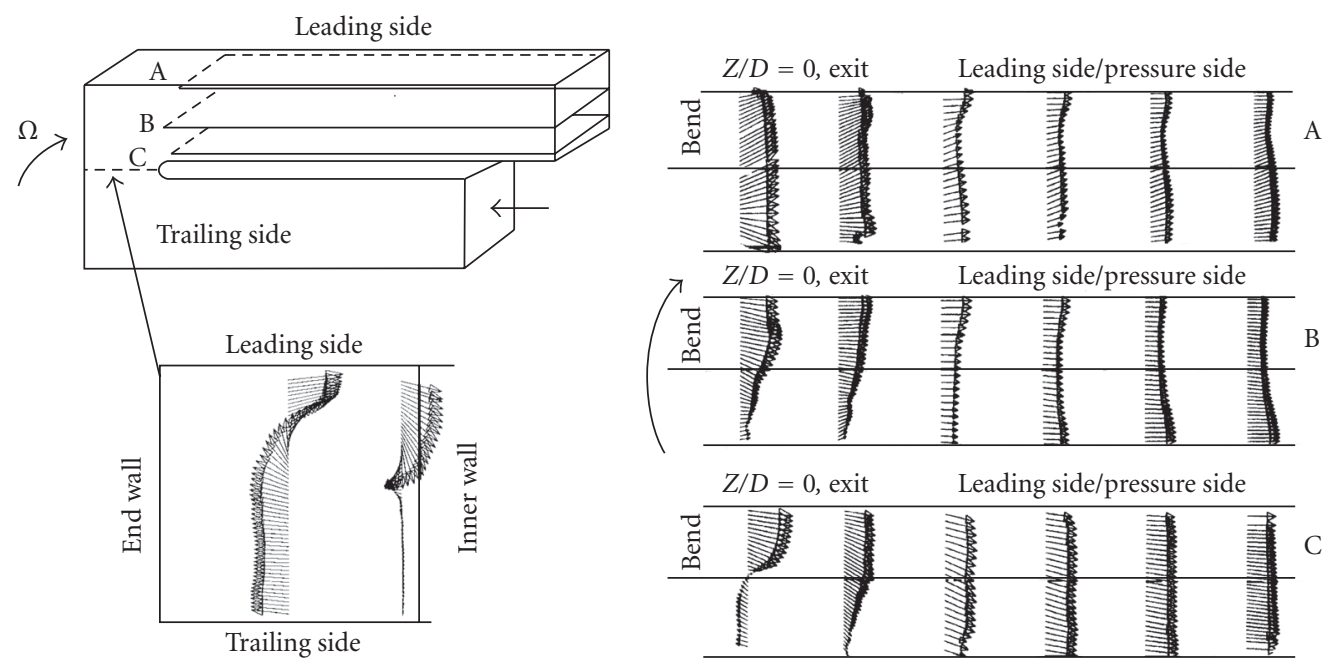

FIGURE 2: Measured flow development (see Iacovides et al. [5]).

model required approximately twice as much storage and also twice the CPU time per iteration as the EVM model. Moreover, the DSM model required approximately twice as many iterations as the EVM to reach a converged solution.

\section{CASES EXAMINED}

The case examined is that of a U-bend of square cross-section with a ratio between the inner radius of the bend and the duct diameter $D$ on 0.15 . Flows at two Reynolds numbers have been computed, one at 100000 and one at 36000 , both at a rotation number $\left(\Omega D / U_{b l}\right)$ of 0.2 , where $U_{b l}$ is the bulk velocity. Heat transfer calculations have been obtained for a Prandtl number (Pr) of 5.9, in order to match the experimental conditions. The flow domain started three diameters before the bend and extended to eight diameters after the bend exit. The grid employed consisted of $58 \times 58$ grid nodes over the cross-section and 104 planes in the streamwise direction. In our recent study of flows through a stationary U-bend of the same geometry, see Nikas and Iacovides [6], computations with a mesh consisting of $45 \times 86$ grid nodes over the half cross-section and 104 streamwise planes produced the same flow and thermal predictions as a $31 \times 58 \times 104$ mesh. The latter mesh has the same cross-sectional density as the one employed here.

\section{RESULTS AND DISCUSSION}

The measurements in Figure 2 show that the orthogonal rotation has a strong influence on the flow development, within and downstream of the bend. The classical symmetric twovortex structure within the bend is replaced by a single vortex, and after the bend, the flow along the inner wall separates only near the trailing side and accelerates near the leading side. It is well known (see Bo et al. [3]) that in the upstream section, the Coriolis force increases the pressure along the trailing side relative to that of the leading side and also results in the transfer of the faster fluid along the trailing side, leaving the slower fluid along the leading side. Within the bend, as the main flow direction becomes parallel to the axis of rotation, the Coriolis force disappears, which removes the difference in pressure between the leading and trailing sides. Consequently, as the fluid enters the bend, it encounters a favourable pressure gradient along the trailing side and an adverse pressure gradient along the leading side. This in turn causes the single-vortex structure within the bend, seen in Figure 2, with strong flow separation along the leading side. The measurements at the bend exit and also flow visualization (see Kelemenis [19]) indicate that this single vortex is still present at the bend exit, causing the flow to separate only along the trailing side. As can be seen in Figure 3, the computations are able to reproduce the main features of the overall flow development. This is not unexpected because these are not turbulence-driven phenomena. Moreover, the predicted secondary velocity vector plots of Figure 4 also confirm the conclusion that the single vortex observed at the $90^{\circ}$ plane is also present, with almost equal intensity, at the bend exit. Even two diameters after the exit, this vortex is still present, though by this stage, it has lost some of its strength.

Figure 5 provides more detailed comparisons of the axial velocity profiles along the mid-plane and also along planes near the inner and outer walls within and up to two diameters after the bend. Comparisons further downstream have not been included, because they show a close agreement between predictions and measurements. Along the outer wall, the different models used return very similar axial velocity predictions and at the bend exit and downstream (where measurements are available), there is very little difference between predictions and measurements. Along the mid-plane, only small differences appear between the predictions of the low-Re and the two-layer models are still small. At the $90^{\circ}$ location, all models return a more gradual change in velocity across the duct than what is measured. Agreement between predictions and measurements improves at the end exit and the monitoring locations further downstream, but 

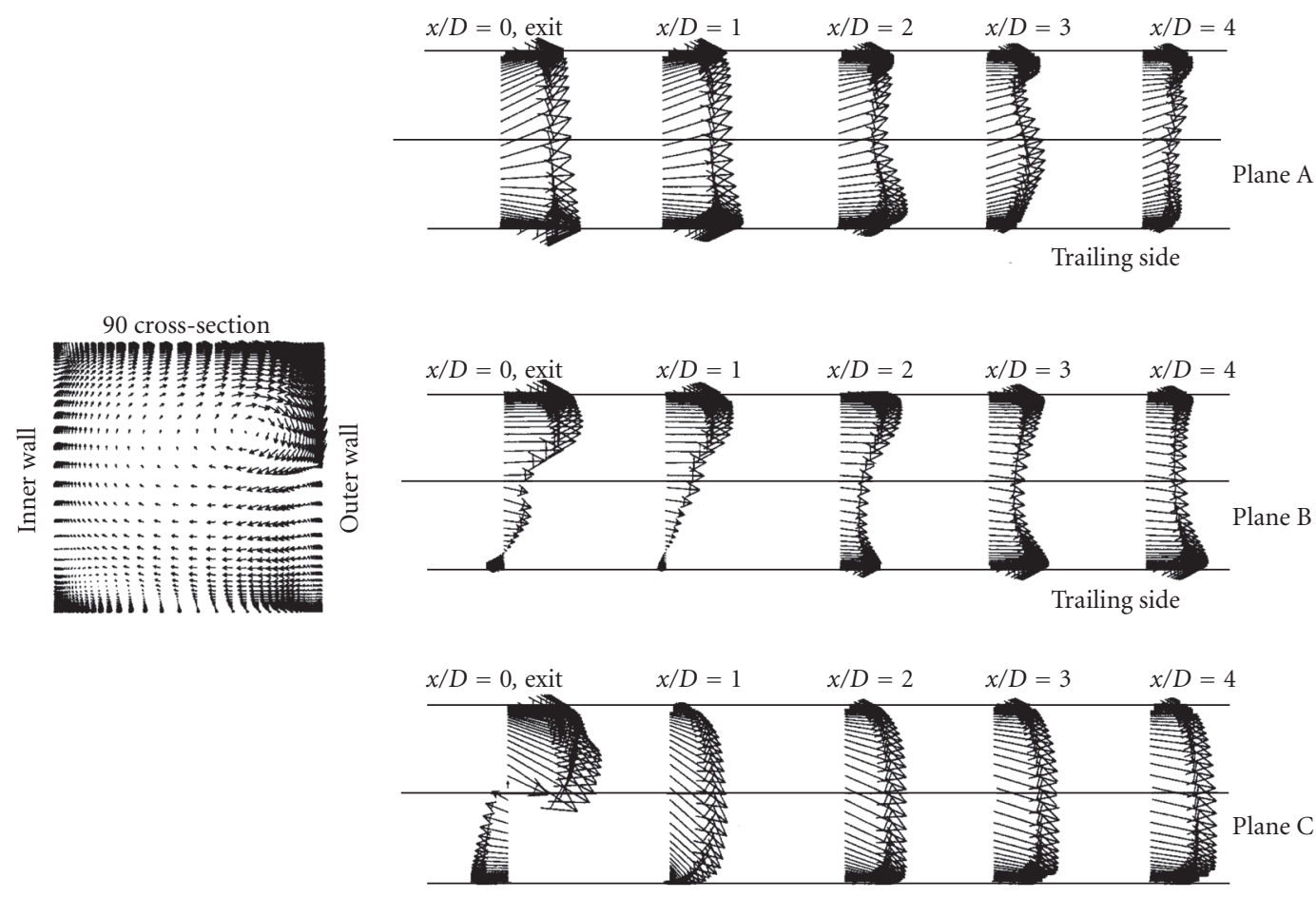

Trailing side

FIgURE 3: Predicted flow development within and downstream of rotating bend, using the low-Re DSM.

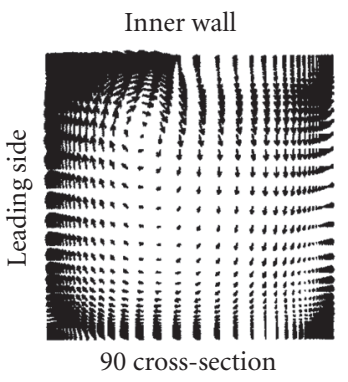

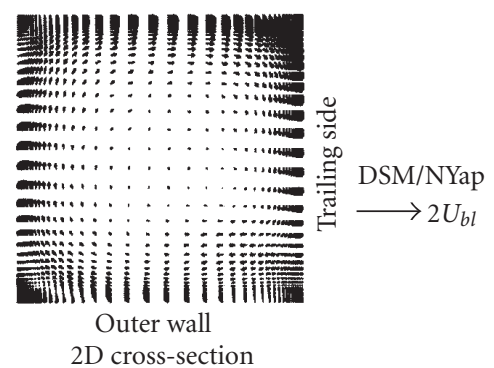

Figure 4: Predicted secondary flow development.

some small differences do persist along the trailing side. Along the inner wall, the comparisons now reveal that the predicted flow development within and just after the U-bend becomes sensitive to the approach adopted for the modelling of near-wall turbulence. Use of two-layer models results in the underprediction of the size of the separation bubble along the trailing side, within the bend, and also to a faster, than measured, recovery just downstream of it. The low-Re models produce mean flow predictions that are close to the measurements. Moreover, the differences between the low-Re effective-viscosity and second-moment closures are relatively minor. These findings are not surprising since along the inner wall, the flow is subjected to an adverse pressure gradient which causes flow separation. It is thus reasonable to expect that the predictions of these more complex flow features will be sensitive to the modelling of near-wall turbulence and also that near-wall models that use the wall distance will be found to be inappropriate. On the whole, both low-Re closures are in good, though not complete, agreement with the measured mean flow development.

The corresponding profile comparisons for the crossduct velocity component, along the direction parallel to the axis of curvature, are shown in Figure 6. The positive values denote the direction from the leading to the trailing side. Along the outer wall, again there are no differences in the predictions of the different models within and just after the bend. The measured cross-duct velocity, however, is somewhat stronger than that computed, especially along the trailing side. At the mid-plane, the data indicate that the magnitude of the cross-duct velocity becomes lower especially after the bend. There are still only minor differences among the computations of the various models, but there are now large 


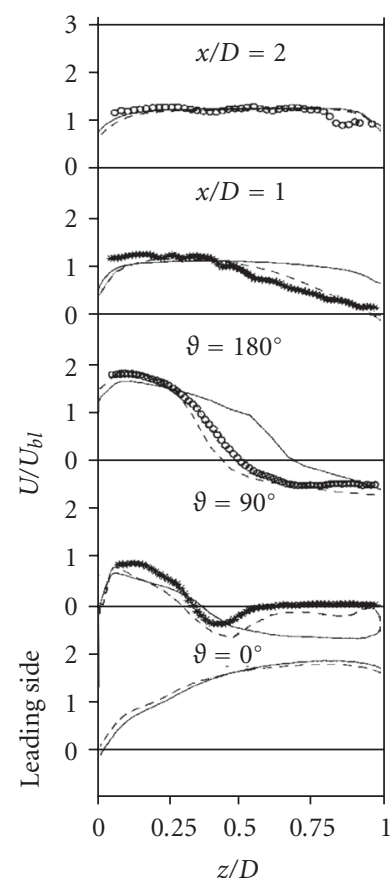

- Two-layer $k-\varepsilon$

- - Low-Re $k-\epsilon$

00000 Experiment (see Iacovides et al. [12])

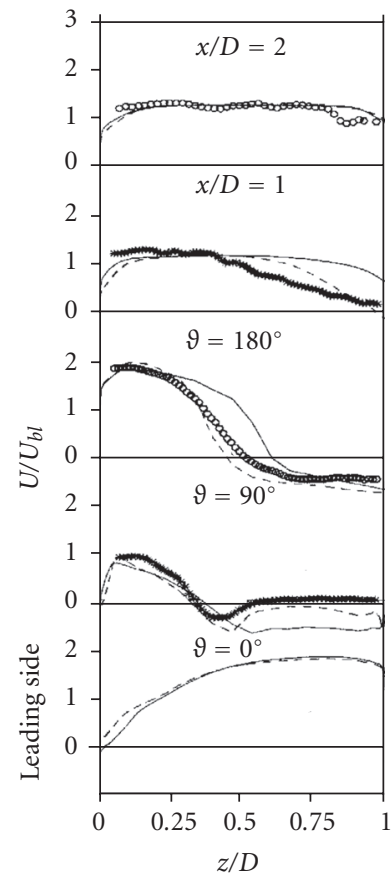

- Two-layer DSM

- - - Low-Re DSM

○oo Experiment (see Iacovides et al. [12])

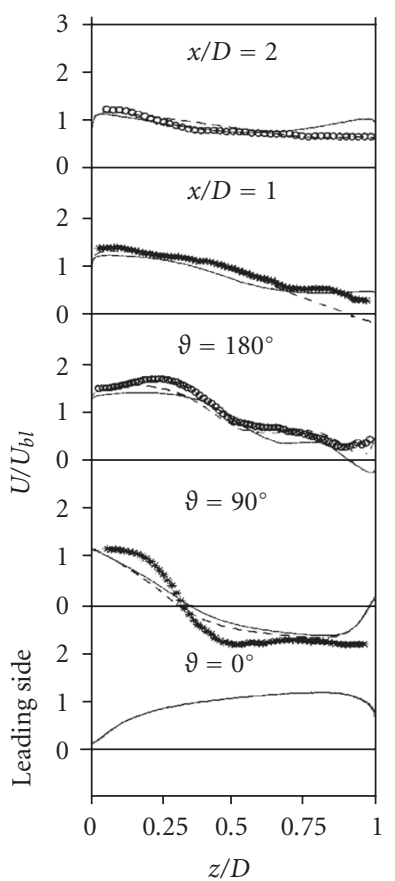

Two-layer $k-\varepsilon$

- - - Low-Re $k-\epsilon$

œ0000 Experiment (see Iacovides et al. [12])

(a)

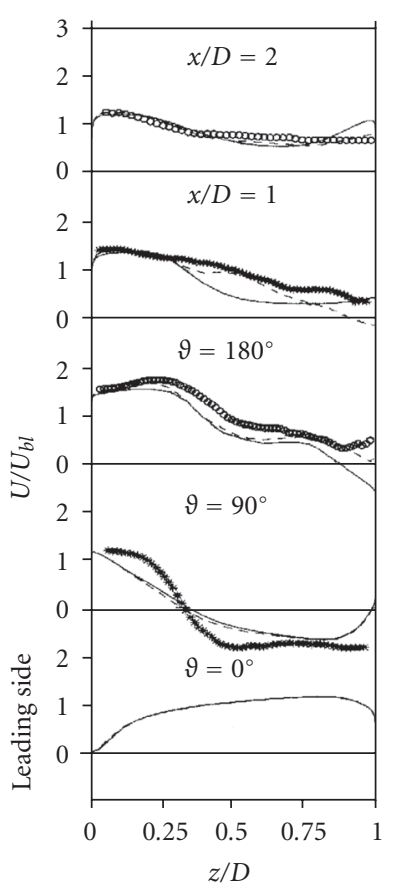

_ Two-layer DSM

- - - Low-Re DSM

œoo0 Experiment (see Iacovides et al. [12])

(a)

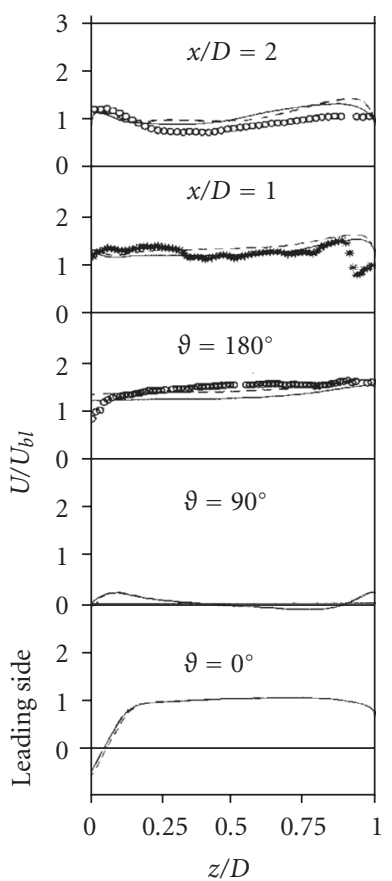

- Two-layer $k-\varepsilon$

- - Low-Re $k-\epsilon$

○oooo Experiment (see Iacovides et al. [12])

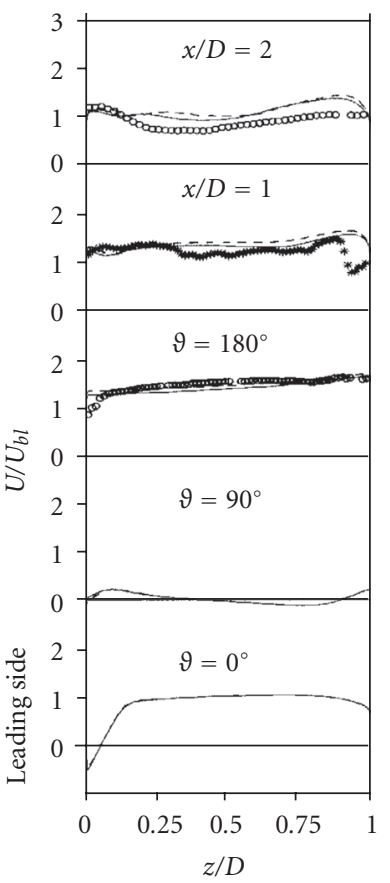

_ Two-layer DSM

- - - Low-Re DSM

§ooo Experiment (see Iacovides et al. [12])

(b)

FIGURE 5: Comparisons between (a) predicted and (b) measured profiles of mean streamwise velocity. The left plot indicates inner plane C, the middle plot indicates mid-plane $\mathrm{B}$, and the right plot indicates outer plane $\mathrm{A}$. 


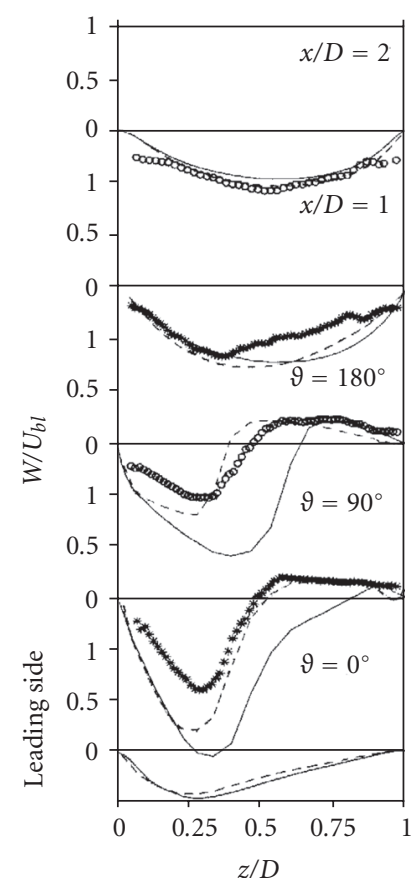

- Two-layer $k-\varepsilon$

- - - Low-Re $k-\epsilon$

ooooo Experiment (see Iacovides et al. [12])

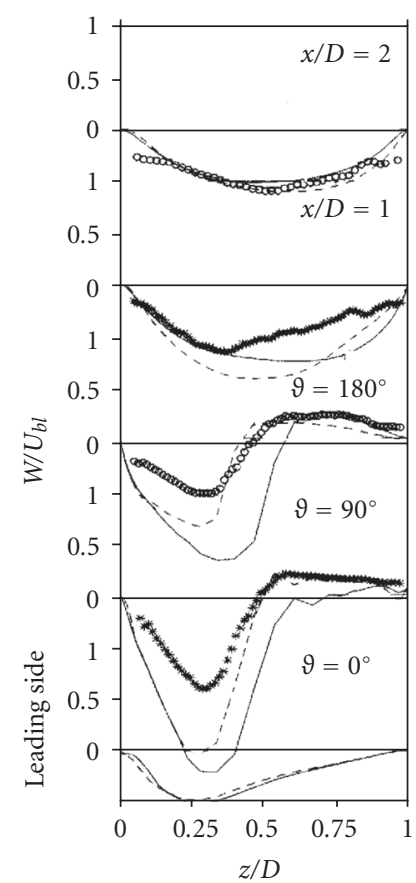

_ Two-layer DSM

- - - Low-Re DSM

oocoo Experiment (see Iacovides et al. [12])

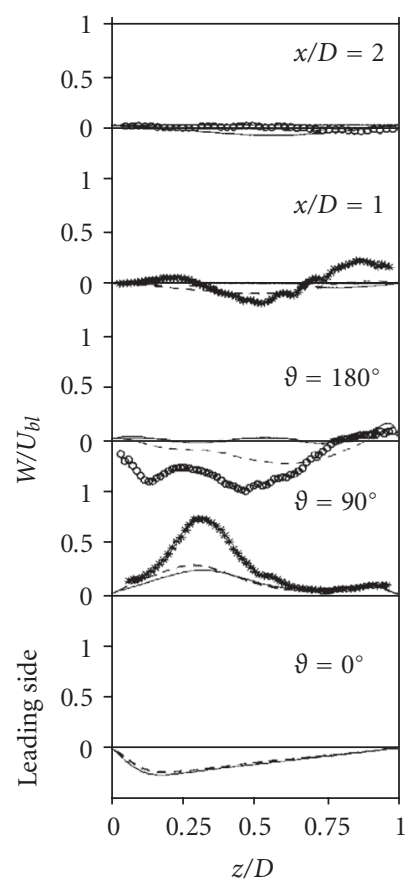

- Two-layer $k-\varepsilon$

- - - Low-Re $k-\epsilon$

ooooo Experiment (see Iacovides et al. [12])

(a)

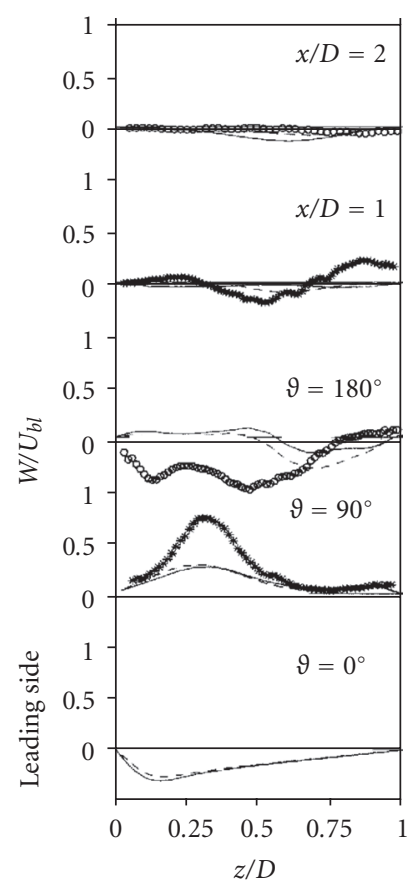

— Two-layer DSM

- - - Low-Re DSM

œoooo Experiment (see Iacovides et al. [12])

)

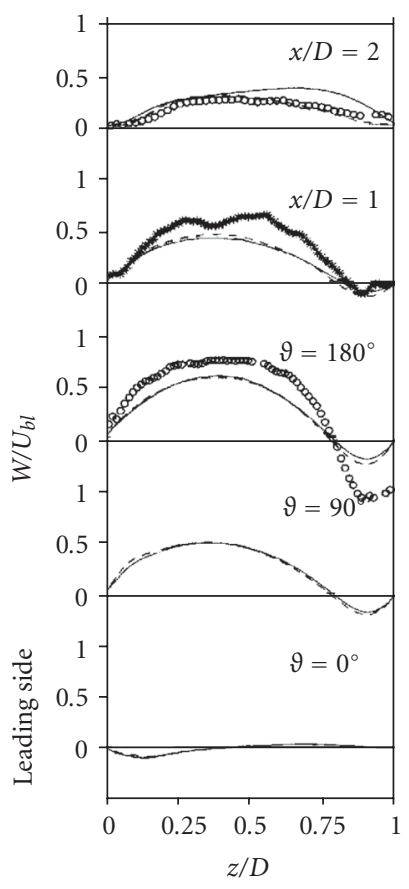

_ Two-layer $k-\varepsilon$

- - - Low-Re $k-\epsilon$

ooooo Experiment (see Iacovides et al. [12])

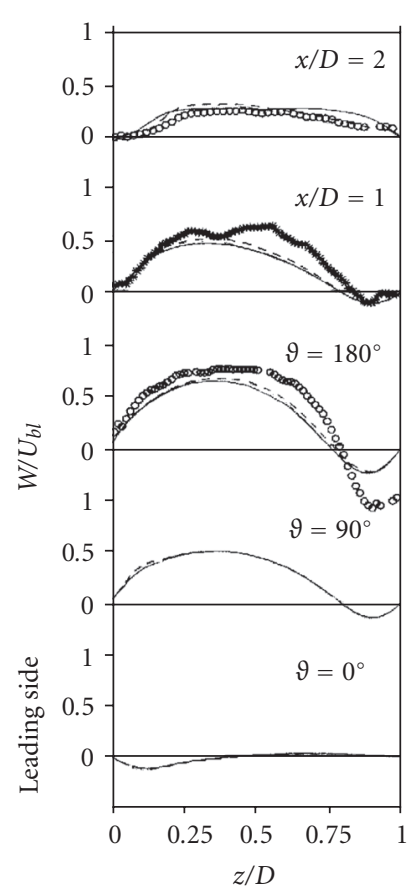

_ Two-layer DSM

- - - Low-Re DSM

œoooo Experiment (see Iacovides et al. [12])

(b)

Figure 6: Comparisons between (a) predicted and (b) measured profiles of mean cross-duct velocity. The left plot indicates inner plane C, the middle plot indicates mid-plane $\mathrm{B}$, and the right plot indicates outer plane $\mathrm{A}$. 
discrepancies with the measured profiles especially within the bend. The cross-duct velocity is underpredicted and at the bend exit, the predictions return also the wrong trends. Along the inner side, as also observed in the comparisons of the axial velocity, there are again large differences between the two-layer and the low-Re predictions, with the latter being closer to the data. The low-Re computations do not deviate from the data by as much as in the mid-plane comparisons, though within the bend, the cross-duct motion near the trailing side is overpredicted. It is thus found that the cross-duct motion is better predicted along the inner and outer walls than at the mid-plane.

Figure 7 shows the corresponding comparisons for the cross-duct component of the turbulence intensity. The introduction of a second-moment closure has some effect on the predicted turbulence field. Within the bend, while all models return turbulence levels that are considerably higher than those of the upstream section, they still underpredict the measured high levels. The distributions produced by the DSM models are closer to those measured than those of the EVM models. Along the near-inner-wall plane, as also noted in the mean velocity comparisons, there are noticeable differences between the predicted intensity levels of the low-Re models and those of the two-layer models. Downstream, as indicated by the comparisons at two diameters after the bend and also further downstream, not shown here due to space limitations, the measured turbulence levels become considerably lower and are well predicted by all the models tested. Moreover, the above comments are also largely applicable to the axial component of the turbulence intensity, for which, again due to space limitations, comparisons are not presented.

Finally, the assessment of the models tested is completed through the comparisons of the axial evolution of the sideaveraged Nusselt number, shown in Figure 8. Here, the comparisons produced by Nikas and Iacovides [6] for the corresponding stationary case have also been included. This has been done in order to highlight the effects of rotation on wall heat transfer and also in order to provide a more complete assessment of the models' capabilities. The measurements show that in the stationary case, the Nusselt number starts to increase inside the U-bend, reaching a maximum value of about twice that of the upstream section, about one diameter after the bend exit and then it starts to gradually fall. Along the trailing side, rotation raises somewhat the Nusselt number levels, especially within and just after the bend, but does not change the overall variation. Along the leading side on the other hand, the effects of rotation are stronger. First, there is a much sharper rise in the Nusselt number at the bend entry where, as noted earlier, the interaction between the Coriolis and curvature forces leads to the formation of a stronger vortex. Then over the second half of the bend and also up to one and a half diameters after the exit, there is a considerable reduction in wall heat transfer in comparison to both the stationary case and also the trailing side. This difference in the thermal behaviour of the two sides is certainly consistent with the measured variation in the cross-duct turbulence intensity between the leading and trailing sides shown in Figure 7. For the stationary case, the comparisons indicate that both the two-layer models produce a reasonable Nusselt number variation within and after the bend, while as far as the low-Reynolds-number models are concerned, only the low-Re DSM with the differential Yap term produces reasonable Nusselt number predictions. In the rotating case, along the trailing side, again none of the computations is in complete agreement with the measurements, but the predictions of the low-Re DSM with the NYap are closest to the data, returning the correct value and location of the maximum Nusselt number after the bend. Even this model, however, underpredicts heat transfer levels at the bend exit and also produces a reduction in wall's heat transfer after the first two diameters which is too gradual in comparison to the data. The low-Re DSM with the standard Yap term, in addition to the predictive deficiencies displayed by the version with the differential Yap term NYap, also overpredicts the peak heat transfer levels after the bend, while the zonal DSM underpredicts the peak heat transfer levels. The low-Re EVM models also underpredict heat transfer levels at the bend entry and within the bend, while the zonal EVM displays predictive deficiencies after the bend. Along the leading side, while all models successfully reproduce the sharp increase in Nusselt number at the bend entry, they fail to predict the drop in Nusselt number over the second half of the bend. This predictive deficiency is even more prominent in the DSM computations, though these models do come closer to predicting the correct recovery further downstream. Clearly, the turbulence models find it very difficult to return the measured thermal behaviour, over the side along which the flow is most complex. Another point worth bearing in mind is that because we are here dealing with a fluid with a Prandtl number greater than one, the thickness of the conduction sublayer, within which the turbulent heat flux becomes negligible in comparison to heat conduction, is considerably thinner than the hydrodynamic viscous sublayer. The Nusselt number is thus more strongly influenced by the turbulence field when in fluids with higher Prandtl number values. Consequently, the failure of turbulence models to return the correct near-wall turbulence field will have a greater detrimental effect on the thermal predictions. One positive outcome of these comparisons is that in the case of the low-Re DSM, the introduction of the differential form of the Yap term, which does not require the use of the wall distance, improves heat transfer predictions.

\section{CONCLUDING REMARKS}

The heat- and fluid-flow computations carried out and the resulting comparisons lead to the following conclusions about the effectiveness of the EVM and DSM models tested in reproducing the flow and thermal development in rotating U-bends. The main flow features generated by the combined presence of rotation and curvature are well reproduced by all models. The detailed mean flow development within and just after the bend is better reproduced by low-Re rather than by two-layer models. Turbulence levels within the rotating 

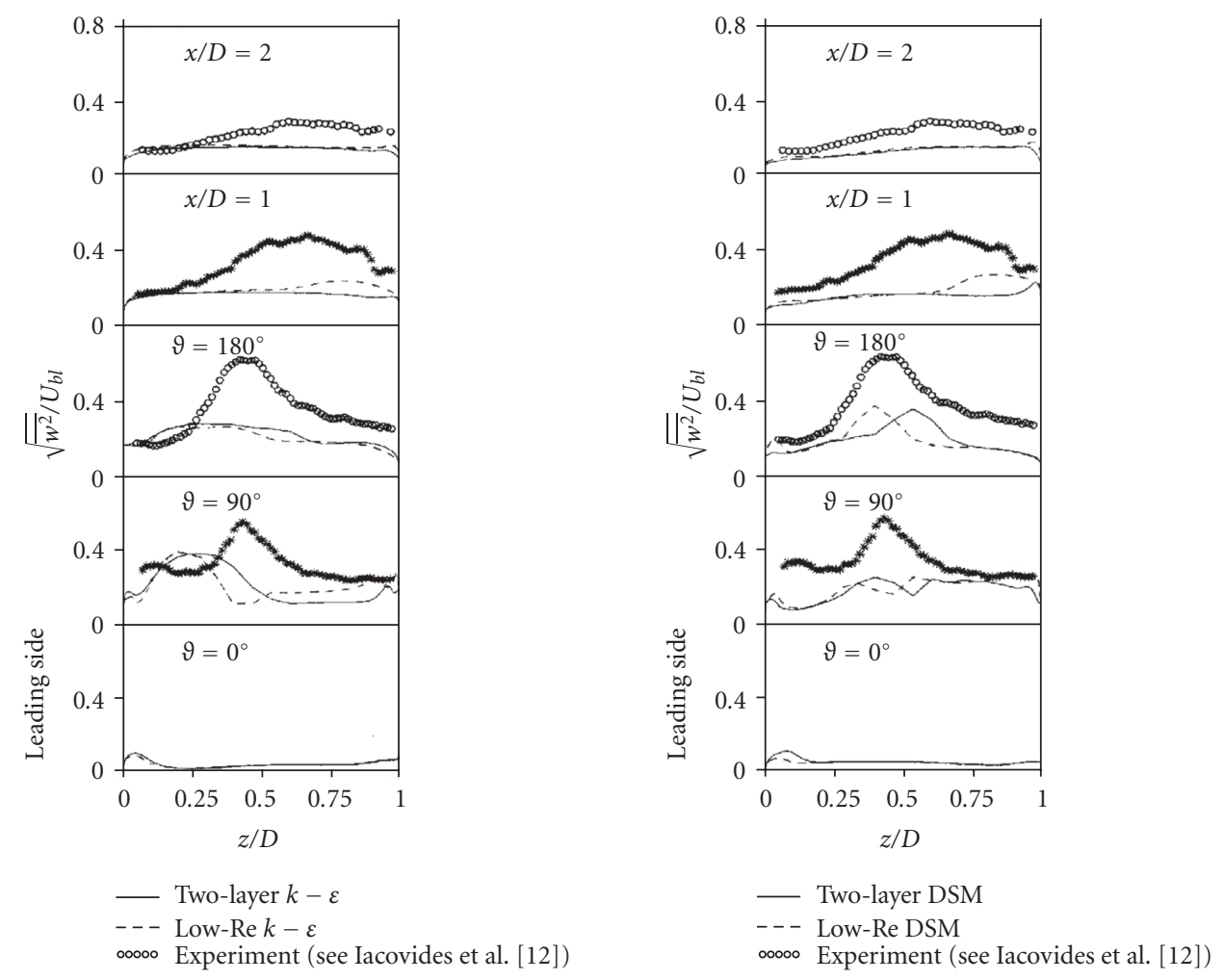

(a)
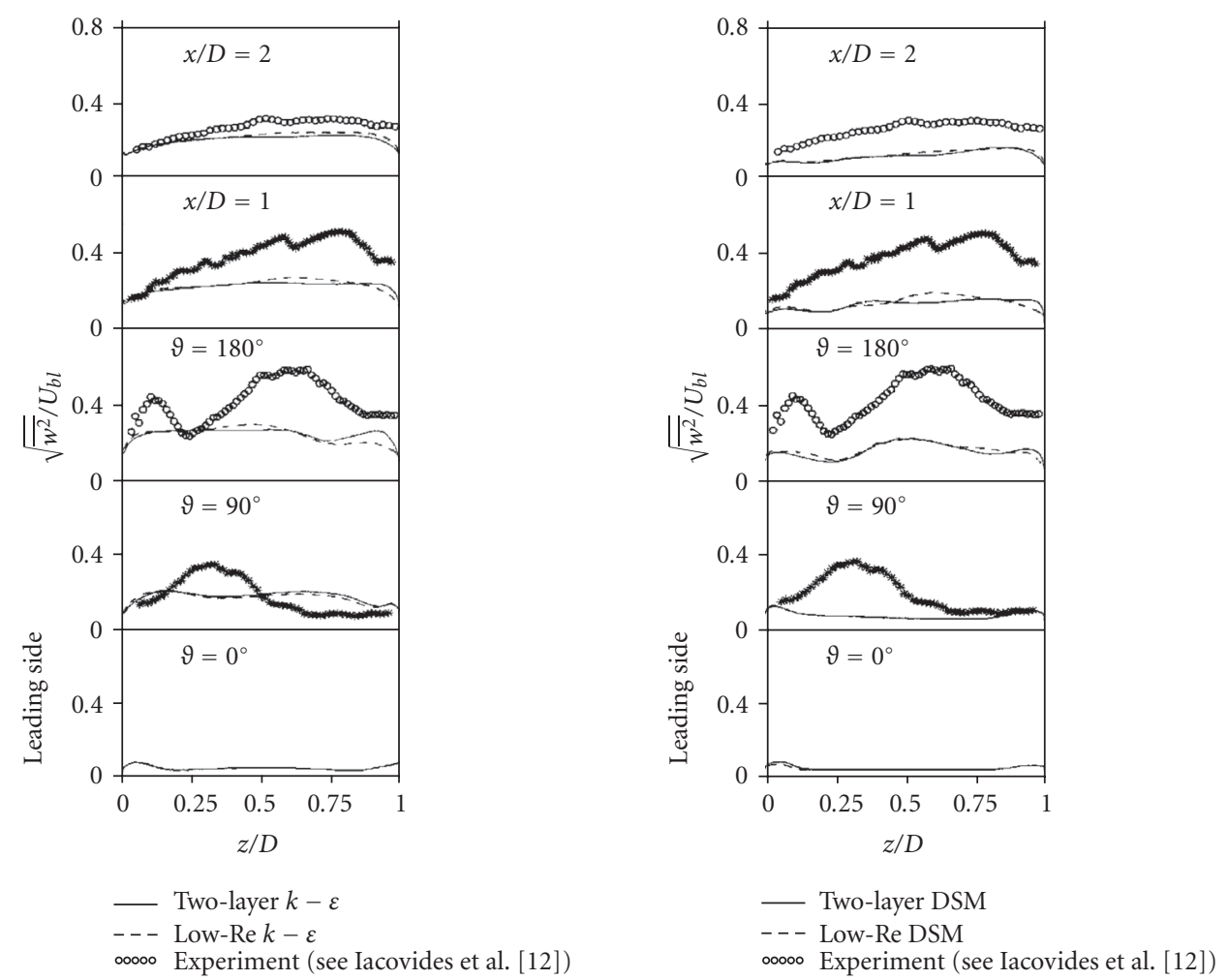

(b)

FIGURE 7: Comparisons between predicted and measured profiles of cross-duct turbulence intensity: (a) inner plane C and (b) mid-plane B. 


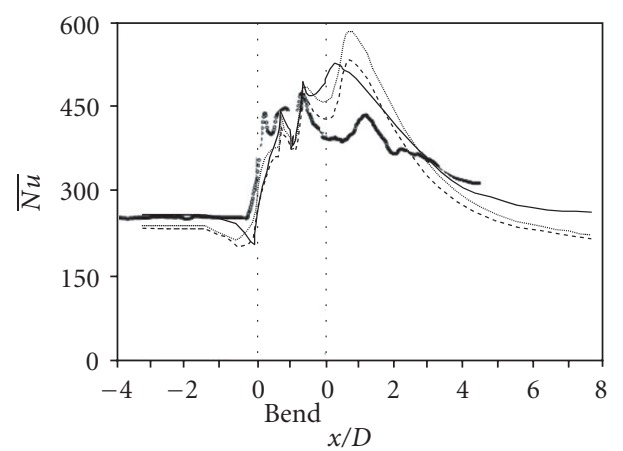

_ Two-layer $k-\varepsilon$

..... Low-Re $k-\varepsilon$

- - L Low-Re $k-\varepsilon /$ NYap

ooooo Experiment (see Iacovides et al. [5])

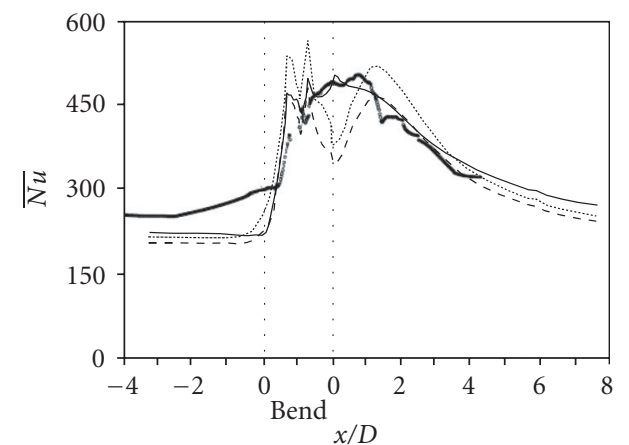

_ Two layer $k-\varepsilon$

..... Low-Re $k-\varepsilon$

- - - Low-Re $k-\varepsilon /$ NYap

ooooo Experiment (see Iacovides et al. [5])

(b)

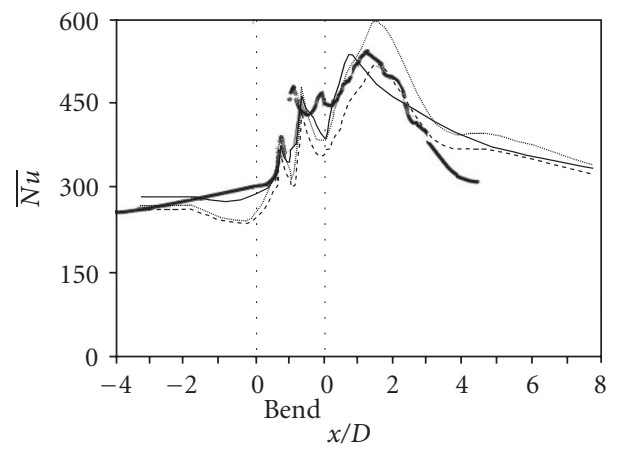

— Two-layer $k-\varepsilon$

…. Low-Re $k-\varepsilon$

- - - Low-Re $k-\varepsilon /$ NYap

ooooo Experiment (see Iacovides et al. [5])

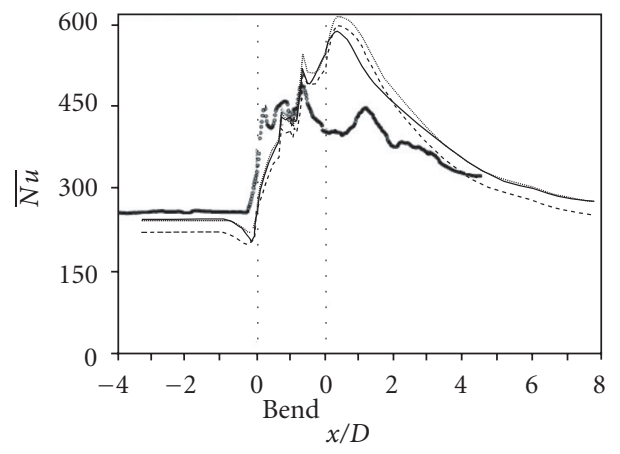

— Two-layer DSM

Low-Re DSM

- - - Low-Re DSM / NYap

oooo Experiment (see Iacovides et al. [5])

(a)

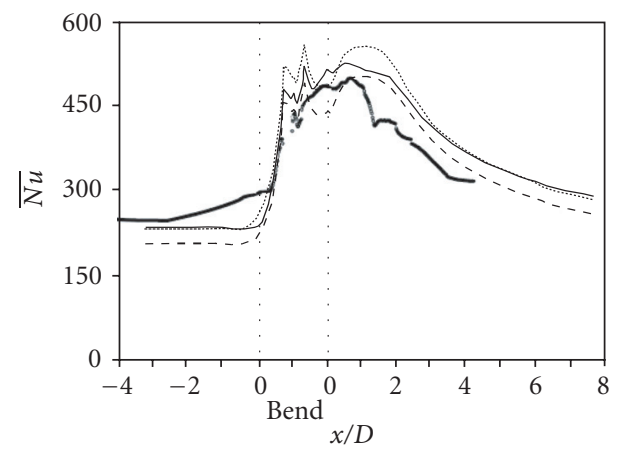

- Two-layer DSM

… Low-Re DSM

- - - Low-Re DSM / NYap

ooooo Experiment (see Iacovides et al. [5])

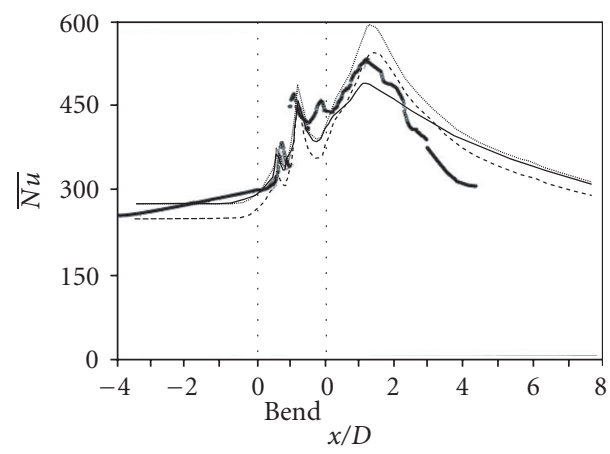

$\begin{array}{ll} & \text { Two-layer DSM } \\ \ldots . . . & \text { Low-Re DSM } \\ --- & \text { Low-Re DSM / NYap } \\ \text { 00000 } & \text { Experiment (see Iacovides et al. [5]) }\end{array}$

(c)

FigURE 8: Comparisons of the axial variation of the side-averaged Nusselt number along the flat wall for the stationary and rotating cases. $\operatorname{Re}=36000, \operatorname{Pr}=5.9$, and $\operatorname{Ro}=0.2$. (a) The leading side, (b) the stationary case, and (c) the trailing side. 
U-bend are underpredicted, but DSM models produce a more realistic distribution. Along the leading side, all models overpredict heat transfer levels just after the bend and along the trailing side, the heat transfer predictions of the low-Re DSM with the differential Yap term are reasonably close to the measurements. This model has also been found to lead to reasonable thermal predictions in a stationary U-bend of the same geometry. The introduction of the differential form of the length-scale correction term (NYap) tends to improve the heat transfer predictions of both low-Re models.

\section{REFERENCES}

[1] S. V. Ekkad and J. C. Han, "Local heat transfer distributions near a sharp $180^{\circ}$ turn of a two-pass smooth square channel using a transient liquid crystal image technique," Journal of Flow Visualization and Image Processing, vol. 2, no. 3, pp. 285297, 1995.

[2] D. L. Rigby, A. A. Ameri, and E. Steinthorssen, "Internal passage heat transfer prediction using multiblock grids and a kw turbulence model," in ASME TURBO EXPO '96, Birmingham, UK, June 1996, ASME Paper, 96-GT-188.

[3] T. Bo, H. Iacovides, and B. E. Launder, "Convective discretization schemes for the turbulence transport equations in flow predictions through sharp U-bends," International Journal of Numerical Methods for Heat \& Fluid Flow, vol. 5, no. 1, pp. 33-48, 1995.

[4] H. Iacovides, B. E. Launder, and H. Y. Li, "The computation of flow development through stationary and rotating U-ducts of strong curvature," International Journal of Heat and Fluid Flow, vol. 17, no. 1, pp. 22-33, 1996.

[5] H. Iacovides, D. C. Jackson, G. I. Kelemenis, B. E. Launder, and Y. M. Yuan, "Experiments on local heat transfer in a rotating square-ended U-bend," International Journal of Heat and Fluid Flow, vol. 20, no. 3, pp. 302-310, 1999.

[6] K.-S. P. Nikas and H. Iacovides, "The computation of flow and heat transfer through square-ended U-bends, using lowReynolds-number models," International Journal of Numerical Methods for Heat \& Fluid Flow, vol. 14, no. 3, pp. 305-324, 2004.

[7] H. Iacovides and M. Raisee, "Recent progress in the computation of flow and heat transfer in internal cooling passages of turbine blades," International Journal of Heat and Fluid Flow, vol. 20, no. 3, pp. 320-328, 1999.

[8] M. Wolfshtein, "The velocity and temperature distribution in one-dimensional flow with turbulence augmentation and pressure gradient," International Journal of Heat and Mass Transfer, vol. 12, no. 3, pp. 301-318, 1969.

[9] B. E. Launder and B. I. Sharma, "Application of the energydissipation model of turbulence to the calculation of flow near a spinning disc," Letters in Heat and Mass Transfer, vol. 1, no. 2, pp. 131-137, 1974.

[10] H. Iacovides and B. E. Launder, "The computation of convective heat transfer in a $180^{\circ}$ pipe bend," in Proc. ICHMT International Symposium on Heat Transfer in Turbomachinery, Athens, Greece, August 1992.

[11] H. Iacovides and P. Toumpanakis, "Turbulence modelling of flow in axisymmetric rotor-stator systems," in Proc. IAHR 5th International Symposium on Refined Flow Modelling and Turbulence Measurements, Paris, France, September 1993.

[12] H. Iacovides, D. C. Jackson, B. E. Launder, and Y. M. Yuan, "Flow measurements in stationary and orthogonally rotating smooth U-bend with rectangular outer wall," Sponsors Report for ABB, EDF \& EGT, Department of Mechanical En- gineering, University of Manchester Institute of Science and Technology, Manchester, UK, 1996.

[13] H. Iacovides and M. Raisee, "Computation of flow and heat transfer in two-dimensional rib-roughened passages, using low-Reynolds-number turbulence models," International Journal of Numerical Methods for Heat \& Fluid Flow, vol. 11, no. 2, pp. 138-155, 2001.

[14] M. M. Gibson and B. E. Launder, "Ground effects on pressure fluctuations in the atmospheric boundary layer," Journal of Fluid Mechanics, vol. 86, pp. 491-511, 1978.

[15] C. R. Yap, Turbulent heat and momentum transfer in recirculating and impinging flows, Ph.D. thesis, Department of Mechanical Engineering, Faculty of Technology, University of Manchester, Manchester, UK, 1987.

[16] T. J. Craft, "Prediction of heat transfer in turbulent stagnation flow with a new second-moment closure," in Proc. 2nd International Conference on Turbulent Heat Transfer, vol. 2, pp. 4.15-4.25, Manchester, UK, 1998.

[17] C. M. Rhie and W. L. Chow, "Numerical study of the turbulent flow past an airfoil with trailing edge separation," AIAA Journal, vol. 21, no. 11, pp. 1525-1532, 1983.

[18] H. Iacovides, "The computation of turbulent flow through stationary and rotating U-bends with rib-roughened surfaces," International Journal for Numerical Methods in Fluids, vol. 29, no. 7, pp. 865-876, 1999.

[19] G. I. Kelemenis, Flow and local thermal measurements in stationary and rotating cooling passages of complex geometries, Ph.D. thesis, Faculty of Technology, Mechanical Engineering Department, University of Manchester Institute of Science and Technology, Manchester, UK, 1999. 

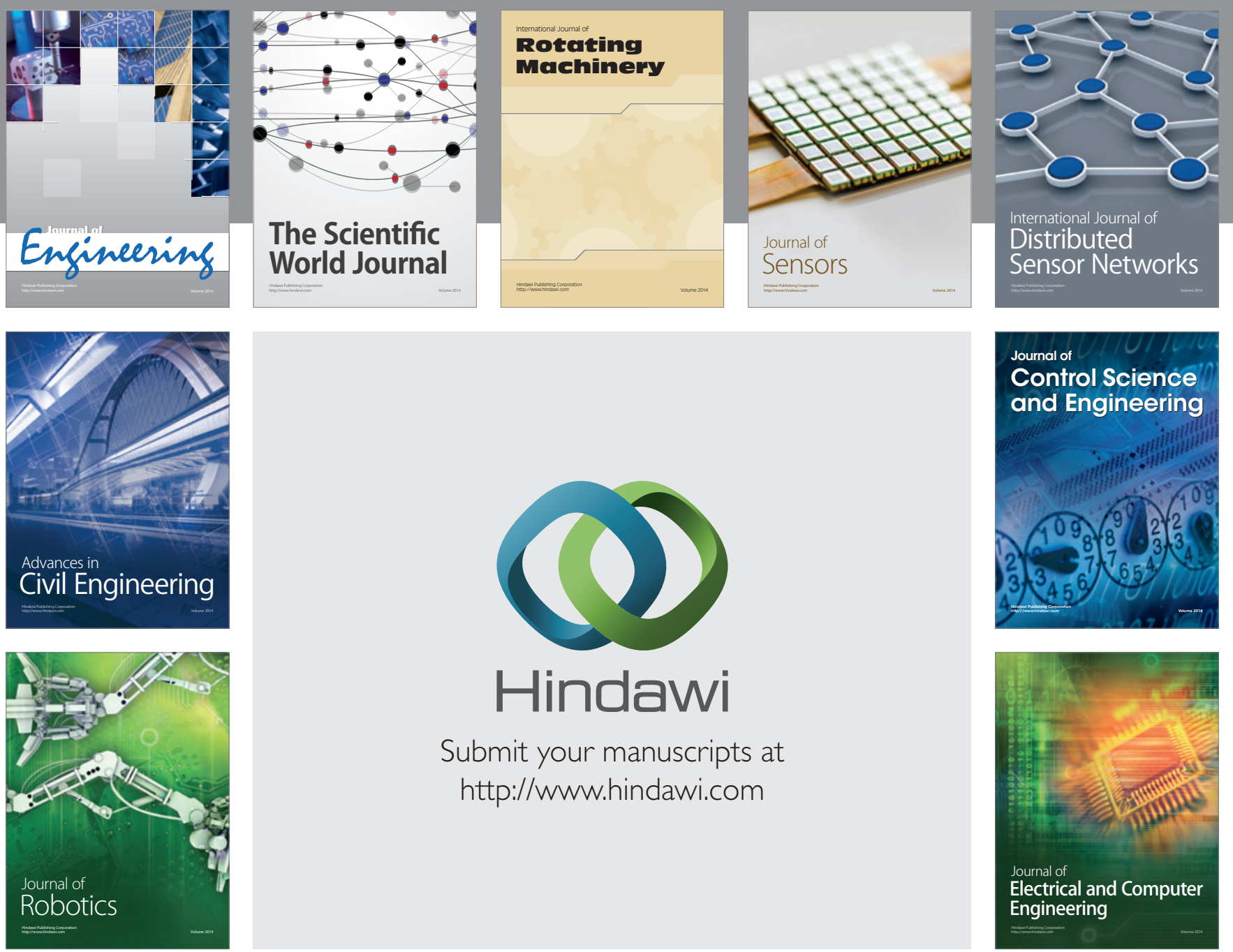

Submit your manuscripts at

http://www.hindawi.com
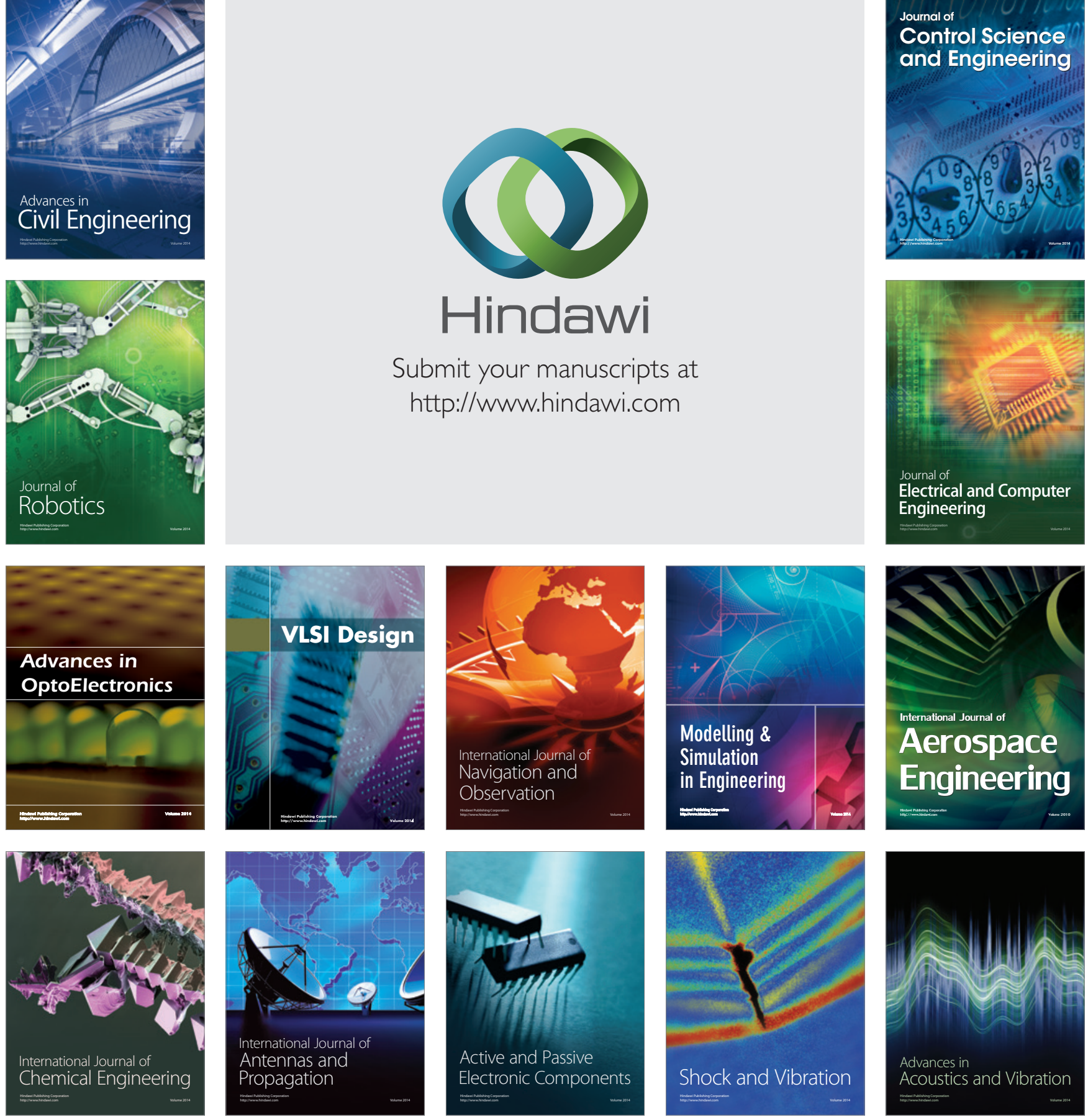